\title{
Fibrinogen-to-albumin ratio predicts long-term outcomes for patients with ST-elevation myocardial infarction and multivessel disease: A prospective observational cohort study
}

\author{
GAO LIU $^{1 *}$, CHUAN-MIN FAN $^{1 *}$, HAO GUO $^{2}$, WEI-NA FAN ${ }^{1},{\text { MING-LIANG } \text { LI }^{3} \text { and GUO-XIONG CUI }}^{4}$ \\ ${ }^{1}$ Department of Cardiology, Xianyang Central Hospital, Xianyang, Shaanxi 712000; ${ }^{2}$ Department of Cardiology, \\ Affiliated Hospital of Yan'an University, Yan'an, Shaanxi 716000; ${ }^{3}$ Department of Cardiology, \\ Hanzhong People's Hospital, Hanzhong, Shaanxi 723000; ${ }^{4}$ Department of Cardiology, \\ Yan'an City Hospital of Traditional Chinese Medicine, Yan'an, Shaanxi 716000, P.R. China
}

Received November 20, 2019; Accepted April 21, 2020

DOI: $10.3892 /$ etm.2021.9896

\begin{abstract}
The fibrinogen-to-albumin ratio index (FAR) is a valuable tool reflecting the systemic inflammation level and associated with the severity of coronary artery disease. However, the utility of the FAR in predicting the long-term prognosis of patients with ST-elevation myocardial infarction (STEMI) and multivessel disease has remained to be determined. A total of 424 patients diagnosed with STEMI and multivessel disease were recruited for the present study. They were given emergent percutaneous coronary intervention treatment and then completed a follow-up for primary (all-cause mortality) and secondary endpoints (major adverse cardiac events, including MI, stroke, emergent revascularization and rehospitalization due to heart failure). The association between FAR and the Synergy Between Percutaneous Coronary Intervention with Taxus and Cardiac Surgery (SYNTAX) score was investigated, while receiver operating characteristic curve analysis was adopted to assess the ability of the FAR to predict long-term outcomes. The long-term survival of high and low FAR groups was compared by drawing Kaplan-Meier survival curves. Multivariate Cox regression analysis was adopted to evaluate the risk factors of primary and secondary endpoints. The FAR was revealed to have a linear correlation with the SYNTAX score $(y=0.022 x+17.737 ; \mathrm{P}=0.015)$. Furthermore,
\end{abstract}

Correspondence to: Dr Guo-Xiong Cui, Department of Cardiology, Yan'an City Hospital of Traditional Chinese Medicine, 6th Floor, Internal Medicine Building, No. 26 Xuanyuan Avenue, New District, Yan'an, Shaanxi 716000, P.R. China

E-mail: 1550878597@qq.com

*Contributed equally

Key words: fibrinogen-to-albumin ratio index, ST-elevation myocardial infarction, all-cause death, cardiac death, Synergy Between Percutaneous Coronary Intervention with Taxus and Cardiac Surgery score, long-term outcomes the FAR was a significant predictor of all-cause death with a cut-off value of 128.4 (area under the curve, 0.832; $\mathrm{P}<0.001$ ). A significant difference was determined between the high FAR group and the low FAR group in terms of the proportion of patients with the primary endpoint $(\mathrm{P}<0.001)$ and secondary endpoint $(\mathrm{P}=0.001)$. It was demonstrated that the FAR was an independent risk factor for all-cause death of patients with STEMI and multivessel disease (hazard ratio, 1.029; 95\% CI: 1.020-1.037; $\mathrm{P}<0.001)$. In summary, the FAR is a valuable biomarker associated with STEMI and may be useful in the prediction of the long-term prognosis of patients with STEMI and multivessel disease.

\section{Introduction}

Among all patients with acute coronary syndrome (ACS), $\sim 50 \%$ suffer from atherosclerosis involving multiple vessels or the entire coronary tree (1). Compared with single-vessel disease, ST-elevation myocardial infarction (STEMI) with multivessel disease is associated with a worse prognosis (2). The results of the Abciximab Intracoronary versus intravenously Drug Application in STEMI demonstrated that although multivessel coronary artery disease (CAD) is not associated with reduced success of reperfusion, multivessel disease is associated with a high risk of adverse clinical outcomes (2). Treatment strategies for STEMI and multivessel disease remain controversial, including percutaneous coronary intervention (PCI) for the culprit vessel or non-culprit vessels, as well as the time-point (3). A useful tool is required for evaluating the condition and predicting the prognosis to aid clinical judgment. The Synergy Between Percutaneous Coronary Intervention with Taxus and Cardiac Surgery (SYNTAX) scoring system is the most widely used tool to determine the extent and severity of CAD. The SYNTAX score is positively correlated with all-cause mortality of patients with CAD after PCI (4). However, the SYNTAX scoring system is complex and there is currently limited data on the effectiveness of SYNTAX score in predicting the prognosis of STEMI patients with multivessel disease. 
The fibrinogen-to-albumin ratio (FAR) is the ratio between serum fibrinogen levels and serum albumin. Being part of the inflammatory reaction and processes associated with thrombosis, fibrinogen is a biomarker for the risk of stroke and MI (5). At the same time, hypoalbuminemia is also associated with severe MI and heart failure (6). The FAR is a valuable biomarker in the prediction of the prognosis of multiple tumors, including esophageal squamous cell carcinoma (7), non-small cell lung cancer (8), colorectal cancer (9) and gallbladder cancer (10). A previous study proved that the FAR is significantly associated with the SYNTAX score and could predict the severity of CAD in patients with STEMI (11). Hence, it was hypothesized in the present study that the FAR may be linked to the long-term prognosis of patients with STEMI and multivessel disease.

\section{Materials and methods}

Study population. The patients were recruited from the emergency department to participate in the present prospective observational cohort study. All patients included were required to fulfill the following criteria: i) Age $\geq 18$ years; ii) diagnosis with STEMI and multiple vessel disease based on coronary angiography; iii) the patient received revascularization treatment of PCI; iv) the patient remained alive during the hospital stay; v) the patient and their family provided written informed consent to participate in the study. The exclusion criteria were as follows: i) Patients who were diagnosed with Non-ST elevation myocardial infarction or unstable angina; ii) patients who were in a coma or unconscious; iii) patients with cancers, severe infections, serious organ dysfunction or other serious diseases; iv) patients and families who were not able to cooperate with the follow-up process.

The 2011 ESC Guidelines for the Management of Acute Myocardial Infarction in Patients Presenting With ST-segment Elevation were followed in the present study (12), according to which STEMI was defined as electrocardiographic ST-segment elevation $>2 \mathrm{~mm}$ in 2 or more contiguous chest leads or $\geq 1 \mathrm{~mm}$ in 2 or more limb leads or new onset of left bundle-branch block, together with chest pain or other typical symptoms and elevated troponin levels $>99$ th percentile (13).

A total of 562 patients (Table I) with STEMI and multiple vessel disease meeting the criteria were recruited for the present study from July 2012 to September 2013 at Xianyang Central Hospital (Xianyang, China). Written informed consent was acquired from the patients and their family. The Medical Ethics Committee of Xianyang Central Hospital (Xianyang, China) approved the present study (no. 20120535). The study design is presented in the flowchart in Fig. 1.

In-hospital treatment. Coronary angiography and interventional therapy were routinely performed. The infarct-related artery only' strategy for PCT was adopted in the present study (14). Successful PCI was defined as coronary angiography indicating residual lumen stenosis of $<10 \%$ and a blood flow classification of Thrombolysis in MI grade 3 (15). In patients with emergency PCI, $300 \mathrm{mg}$ aspirin was administered as soon as possible prior to surgery, and furthermore, oral clopidogrel 300-600 mg or ticagrelor $180 \mathrm{mg}$ was administered orally. Patients were given oral aspirin $100 \mathrm{mg} / \mathrm{day}$ and clopidogrel $75 \mathrm{mg} /$ day (or ticagrelor $90 \mathrm{mg}$ twice daily) for at least 1 year according to the 2011 ESC Guidelines for the Management of Acute Myocardial Infarction in Patients Presenting With ST-segment Elevation (12).

Data collection. Clinical data were collected from the electronic medical record database of Xianyang Central Hospital. The FAR was calculated from serum fibrinogen and albumin concentrations on admission. Plasma fibrinogen levels were detected by the coagulation method using STA CompactMax automatic coagulation analyzer (Diagnostica Stago Ltd.) and high-sensitivity c-reactive protein (hs-CRP) was detected by immunoturbidimetry using an AU5400 automatic biochemical analyzer (Olympus Corporation). Albumin levels and myocardial injury markers, including cardiac troponin $\mathrm{T}$ (cTnT) as well as creatinine kinase (CK)-MB, creatinine, NT-proB-type natriuretic peptide (NT-proBNP), alanine aminotransferase (ALT) and aspartate aminotransferase (AST) were also tested using the Abbott Architect C16000 autoanalyzer (Abbott Laboratories) on admission.

Clinical endpoints. The primary endpoint was defined as all-cause mortality during follow-up and the secondary endpoint was a composite of major adverse cardiac events (MACE), including myocardial infarction (MI), stroke, unscheduled revascularization and rehospitalization due to heart failure.

Follow-up. All patients enrolled were followed up by telephone or visits to the original clinics where they were enrolled every year for a total of 5 years. The parameters determined during follow-up included survival status, recent readmission and other complications. The data of cases with self-dropout or missed contact were considered as censored data.

Statistical analysis. SPSS version 19.0 (IBM Corp) was used for statistical analysis in the present study. The variables were first subjected to a normality of distribution test (Kolmogorov-Smirnov test). Continuous variables following a normal distribution were expressed as the mean \pm standard deviation and categorical variables were presented as proportions, while continuous variables that did not fit a normal distribution were described as the median and interquartile range. Comparison of continuous variables between different groups was performed using one-way analysis of variance and Tukey's multiple-comparisons test of independent samples. The Kruskal-Wallis test and Dunn's multiple-comparisons test were adopted for comparison of different groups for non-normal distributed variables of independent samples. A $\chi^{2}$ test was performed for comparisons of categorical variables. Linear regression analysis was used to examine the correlation between the SYNTAX score and the FAR.

A receiver operating characteristic (ROC) curve analysis was also performed to determine the best cut-off value of the FAR in predicting all-cause death, based on which all patients were divided into a high FAR group and a low FAR group. The Cox proportional hazards model was adopted as the regression method to determine hazard ratios (HR) compare the relative hazard between the high FAR group and the low FAR group. Univariate analysis between covariates and endpoints 
Table I. Demographic characteristics and clinical data of the patients enrolled stratified by the endpoints.

\begin{tabular}{|c|c|c|c|c|}
\hline Variables & $\begin{array}{l}\text { Patients without } \\
\text { endpoints } \\
(n=291)\end{array}$ & $\begin{array}{l}\text { Patients with } \\
\text { primary endpoint } \\
\qquad(\mathrm{n}=38)\end{array}$ & $\begin{array}{l}\text { Patients with } \\
\text { secondary endpoint } \\
\qquad(\mathrm{n}=95)\end{array}$ & P-value \\
\hline \multicolumn{5}{|l|}{ Demographics } \\
\hline Age (years) & $61.5 \pm 15.8$ & $71.5 \pm 10.3^{b}$ & $64.0 \pm 12.7^{\mathrm{b}, \mathrm{c}}$ & $<0.001$ \\
\hline Male sex & $181(62.2)$ & $23(60.5)$ & $57(60.0)$ & 0.921 \\
\hline BMI $\left(\mathrm{kg} / \mathrm{m}^{2}\right)$ & $24.6 \pm 3.2$ & $25.1 \pm 2.7$ & $24.1 \pm 3.3$ & 0.234 \\
\hline Smoking & $108(37.1)$ & $8(21.1)$ & $36(37.8)$ & 0.136 \\
\hline LVEF & $49.6 \pm 10.0$ & $45.6 \pm 11.7$ & $50.4 \pm 9.9$ & 0.065 \\
\hline \multicolumn{5}{|l|}{ Comorbidities } \\
\hline Heart failure & $79(27.1)$ & $19(50.0)^{\mathrm{b}}$ & $16(16.8)^{\mathrm{c}}$ & $<0.001$ \\
\hline Hypertension & $68(23.4)$ & $16(42.1)^{\mathrm{b}}$ & $29(30.5)$ & 0.031 \\
\hline Diabetes mellitus & $46(15.8)$ & $5(13.2)$ & $15(15.8)$ & 0.912 \\
\hline Chronic kidney disease & $13(4.5)$ & $4(10.5)$ & $3(3.2)$ & 0.182 \\
\hline Chronic lung disease & $34(11.7)$ & $3(7.9)$ & $8(8.4)$ & 0.569 \\
\hline Cerebrovascular disease & $17(5.8)$ & $1(2.6)$ & $9(9.5)$ & 0.278 \\
\hline Tumor & $8(2.7)$ & $1(2.6)$ & $3(3.2)$ & 0.976 \\
\hline \multicolumn{5}{|c|}{ Laboratory tests on admission } \\
\hline Peak cTnT (ng/dl) & $4.80(2.90,6.39)$ & $5.43(3.18,6.90)$ & $4.46(2.93,6.17)$ & 0.403 \\
\hline Peak CK-MB (ng/dl) & $552.1(419.8,668)$ & $493.7(379.7,676.6)$ & $593.6(420.3,717.2)$ & 0.183 \\
\hline Creatinine $(\mu \mathrm{mol} / \mathrm{l})$ & $103.1(76.2,131.4)$ & $112.5(72.8,136.0)$ & $94.0(70.6,122.5)$ & 0.190 \\
\hline NT-proBNP (ng/l) & $71.1(27.3,123.1)$ & $91.3(55.7,168.4)$ & $74.2(35.9,123.2)$ & 0.073 \\
\hline $\mathrm{hsCRP}(\mathrm{mg} / \mathrm{l})$ & $21.5(12.9,30.2)$ & $27.1(21.6,36.0)^{\mathrm{b}}$ & $24.0(15.3,32.3)$ & 0.008 \\
\hline $\operatorname{ALT}(\mathrm{U} / \mathrm{l})$ & $14(6,23)$ & $17(9,24)$ & $16(8,23)$ & 0.289 \\
\hline AST (U/l) & $20(11,29)$ & $24(10.5,31.3)$ & $21(11,30)$ & 0.636 \\
\hline Fibrinogen $(\mu \mathrm{g} / \mathrm{ml})$ & $278(216,336)$ & $417(277,492)^{\mathrm{b}}$ & $280(217,349)^{\mathrm{c}}$ & $<0.001$ \\
\hline Albumin (g/dl) & $3.37(3.11,3.69)$ & $3.14(2.73,3.38)^{\mathrm{b}}$ & $3.32(3.09,3.56)^{\mathrm{c}}$ & $<0.001$ \\
\hline SYNTAX score & $20(16,23)$ & $24(20,30)^{\mathrm{b}}$ & $19(16,22)^{\mathrm{c}}$ & $<0.001$ \\
\hline FAR & $81.2(63.4,99.5)$ & $136.6(98.3,156.3)^{\mathrm{b}}$ & $84.0(67.3,106.0)^{\mathrm{c}}$ & $<0.001$ \\
\hline
\end{tabular}

${ }^{a} \mathrm{P}$-value for comparison of the three groups. ${ }^{b} \mathrm{P}<0.05$ vs. patients without endpoints; ${ }^{\mathrm{c}} \mathrm{P}<0.05$ vs. patients with primary endpoint. Values are expressed as the mean \pm standard deviation, $\mathrm{n}(\%)$ or the median (interquartile range). BMI, body mass index; LVEF, left ventricular ejection fraction; cTnT, cardiac troponin T; CK-MB, creatine kinase-MB; NT-proBNP, NT-proB-type natriuretic peptide; hsCRP, high-sensitivity C-reactive protein; ALT, alanine aminotransferase; AST, aspartate aminotransferase; FAR, fibrinogen-to-albumin ratio.

was performed and covariates with $\mathrm{P}<0.10$ were entered into the multivariate analysis. The survival status was compared between the two groups via Kaplan-Meier survival curves and log-rank tests. $\mathrm{P}<0.05$ was considered to indicate statistical significance.

\section{Results}

Demographics and baseline characteristics. A total of 562 patients were enrolled in the current study, with an average age of $(68.3 \pm 20.3$ years $)$, a male/female ratio of $64.9 \%$ (365/562). Among the 562 patients, $=424$ patients completed the 5-year follow-up. During this period, 38 cases of all-cause death occurred, while secondary endpoints were observed in 95 patients, including 41 cases of recurrent MI, 31 cases of unscheduled revascularization, 13 cases of rehospitalization due to heart failure and 10 cases of stroke. The remaining 291 patients did not reach any endpoint.
The demographics and baseline data of patients without endpoints, patients with the primary endpoints and patients with secondary endpoints we compared in Table I. The three groups had similar data in terms of sex ratio, body mass index and smoking status (all $\mathrm{P}>0.05$ ). However, the patients with primary endpoints were significantly older than those in the other two groups $(\mathrm{P}<0.001)$. Furthermore, they had a significantly higher proportion of heart failure $(\mathrm{P}<0.001)$ and hypertension $(\mathrm{P}=0.031)$ than other two groups, but the three groups had no disparity in diabetes mellitus, chronic kidney disease, chronic lung disease, cerebrovascular disease and tumors (All P>0.05). As for laboratory test results on admission, no significant difference was identified among the three groups in peak cTnT, peak CK-MB, creatinine, NT-proBNP, ALT and AST (all P>0.05), while patients with primary or secondary endpoints had significantly higher hs-CRP $(\mathrm{P}<0.001)$ and fibrinogen $(\mathrm{P}<0.001)$ and lower albumin $(\mathrm{P}<0.001)$. 


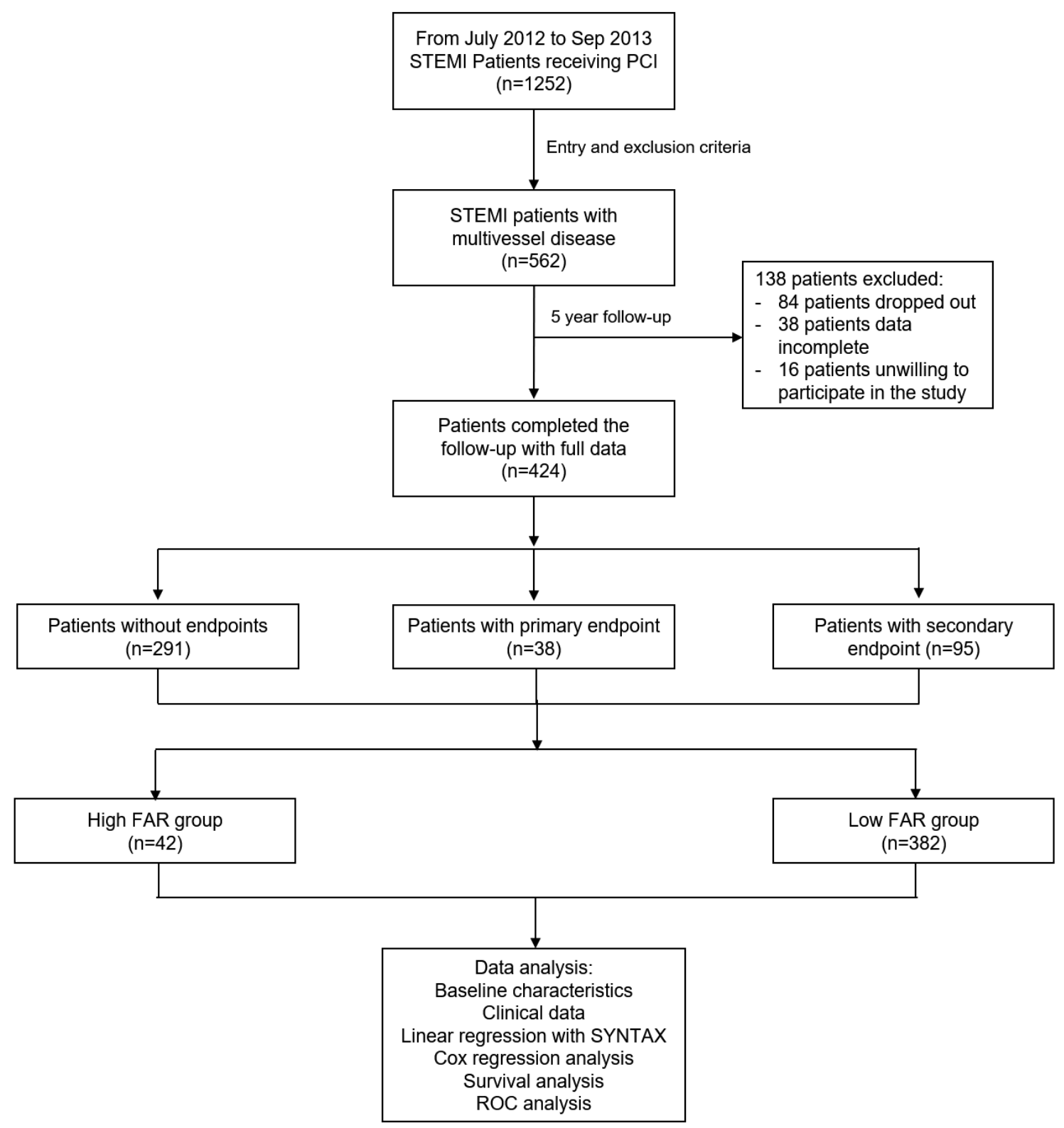

Figure 1. Flowchart depicting the study design. STEMI, ST-elevation myocardial infarction; PCI, percutaneous coronary intervention; FAR, fibrinogen-to-albumin ratio; SYNTAX, Synergy Between Percutaneous Coronary Intervention with Taxus and Cardiac Surgery; ROC, receiver operating characteristic.

Lesions and treatment. The coronary artery lesions and treatments were compared between the different groups (Table II). As for the angiographic findings, a great disparity between patients with and those without endpoints was apparent. The patients with the primary endpoint had a higher number of diseased arteries and this group had a higher proportion of patients with 3-vessel disease, left main CAD and chronic total occlusion (all $\mathrm{P}<0.01$ ). As a consequence, there was also a significant difference in the number of stents used in PCI among the three groups $(\mathrm{P}<0.001)$. However, no significant difference was observed in culprit artery type, use of DES and medication at discharge (all $\mathrm{P}>0.05$ ).

Correlation between SYNTAX score and the FAR. The association between the SYNTAX score and FAR in patients with STEMI and multiple vessel disease was also validated. As presented in Table I, patients with primary outcomes had a significantly higher SYNTAX score and FAR than the other two groups $(\mathrm{P}<0.001)$. The correlation between the SYNTAX and the FAR was then examined by linear regression analysis, which demonstrated that the SYNTAX score had a linear correlation with the FAR (r, 0.118; y, 0.022x+17.737; P=0.015; Fig. 2).

ROC curve analysis. A ROC curve analysis was performed to determine the value of the FAR in the prediction of the long-term outcomes of patients with STEMI and multivessel disease (Fig. 3). It was demonstrated that FAR is significant in predicting all-cause death with an AUC of 0.832 (95\% CI for the AUC, $0.750-0.915 ; \mathrm{P}<0.001)$ at the ideal cut-off value of the FAR determined at 128.4 . The sensitivity was $63.2 \%$ and the specificity was $95.3 \%$.

Primary endpoint. Using the cut-off value of 128.4 , the patients were divided into the high FAR group $(n=42)$ and 
Table II. Lesions and treatment of the patients enrolled stratified by the endpoints.

\begin{tabular}{|c|c|c|c|c|}
\hline Item & $\begin{array}{l}\text { Patients without } \\
\text { endpoints } \\
(n=291)\end{array}$ & $\begin{array}{l}\text { Patients with } \\
\text { primary endpoint } \\
\qquad(\mathrm{n}=38)\end{array}$ & $\begin{array}{c}\text { Patients with } \\
\text { secondary endpoint } \\
(n=95)\end{array}$ & $\mathrm{P}$-value ${ }^{\mathrm{a}}$ \\
\hline \multicolumn{5}{|l|}{ Angiographic findings } \\
\hline Number of diseased arteries & $2.3 \pm 0.5$ & $2.7 \pm 0.4^{\mathrm{b}}$ & $2.2 \pm 0.3^{\mathrm{c}}$ & $<0.001$ \\
\hline$\geq 3$-vessel disease & $104(35.7)$ & $25(65.8)^{\mathrm{b}}$ & $45(47.4)$ & 0.001 \\
\hline LMCA disease & $10(3.4)$ & $6(15.8)^{b}$ & $5(5.3)^{\mathrm{c}}$ & 0.005 \\
\hline Chronic total occlusion & $20(6.9)$ & $10(26.3)^{b}$ & $11(11.6)$ & 0.001 \\
\hline Culprit artery & & & & 0.552 \\
\hline Left anterior descending artery & $124(42.6)$ & $18(47.4)$ & $33(34.7)$ & \\
\hline Circumflex artery & $51(17.5)$ & $8(21.1)$ & $21(22.1)$ & \\
\hline Right coronary artery & $114(39.2)$ & $11(28.9)$ & $40(42.1)$ & \\
\hline Left main coronary artery & $2(0.7)$ & $1(2.6)$ & $1(1.1)$ & \\
\hline \multicolumn{5}{|l|}{ PCI } \\
\hline Number of stents & $2.1 \pm 0.6$ & $2.5 \pm 0.5^{\mathrm{b}}$ & $2.4 \pm 0.3^{\mathrm{b}}$ & $<0.001$ \\
\hline Use of DES & $80(27.5)$ & $15(39.5)$ & $28(29.5)$ & 0.308 \\
\hline \multicolumn{5}{|l|}{ Medication at discharge } \\
\hline Aspirin & $289(99.3)$ & $38(100.0)$ & $94(98.9)$ & 0.805 \\
\hline Clopidogrel & $256(88.0)$ & $33(86.8)$ & $80(84.2)$ & 0.638 \\
\hline Ticagrelor & $35(12.0)$ & $5(13.2)$ & $15(15.8)$ & 0.638 \\
\hline
\end{tabular}

${ }^{\text {a }} \mathrm{P}$-value for comparison of three groups. ${ }^{\mathrm{b}} \mathrm{P}<0.05$ vs. patients without endpoints; ${ }^{\mathrm{c}} \mathrm{P}<0.05$ vs. patients with primary endpoint. Values are expressed as the mean \pm standard deviation or $\mathrm{n}(\%)$.

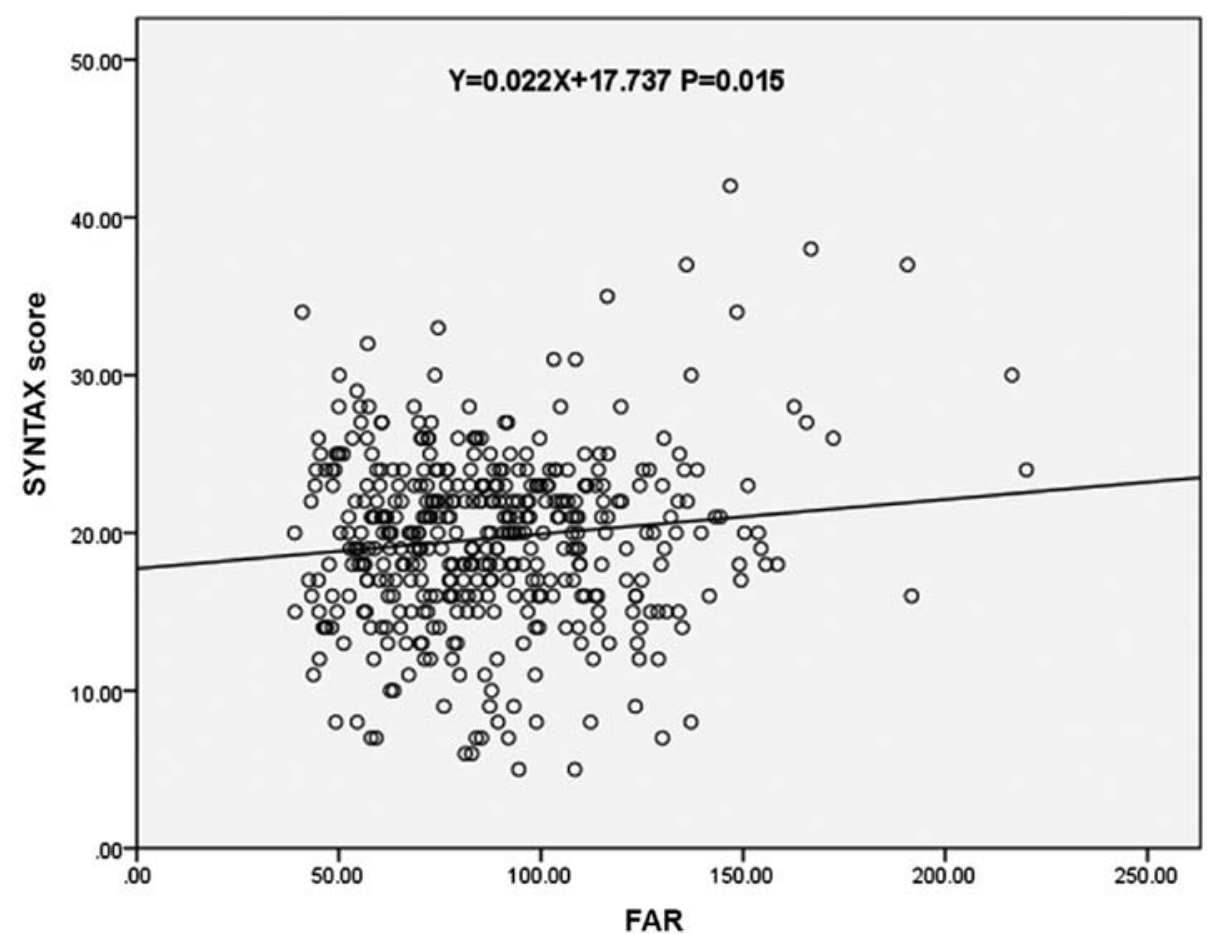

Figure 2. Scatter plot presentation of SYNTAX score and FAR. Linear regression formula: $Y=0.022 X+17.737(r=0.118 ; P=0.015)$. FAR, fibrinogen-to-albumin ratio; SYNTAX, Synergy Between Percutaneous Coronary Intervention with Taxus and Cardiac Surgery.

the low FAR group ( $\mathrm{n}=382)$, and the long-term survival was compared using Kaplan-Meier curves. The results indicated that the two groups had significantly different survival curves. Compared with the low FAR group, the high FAR 


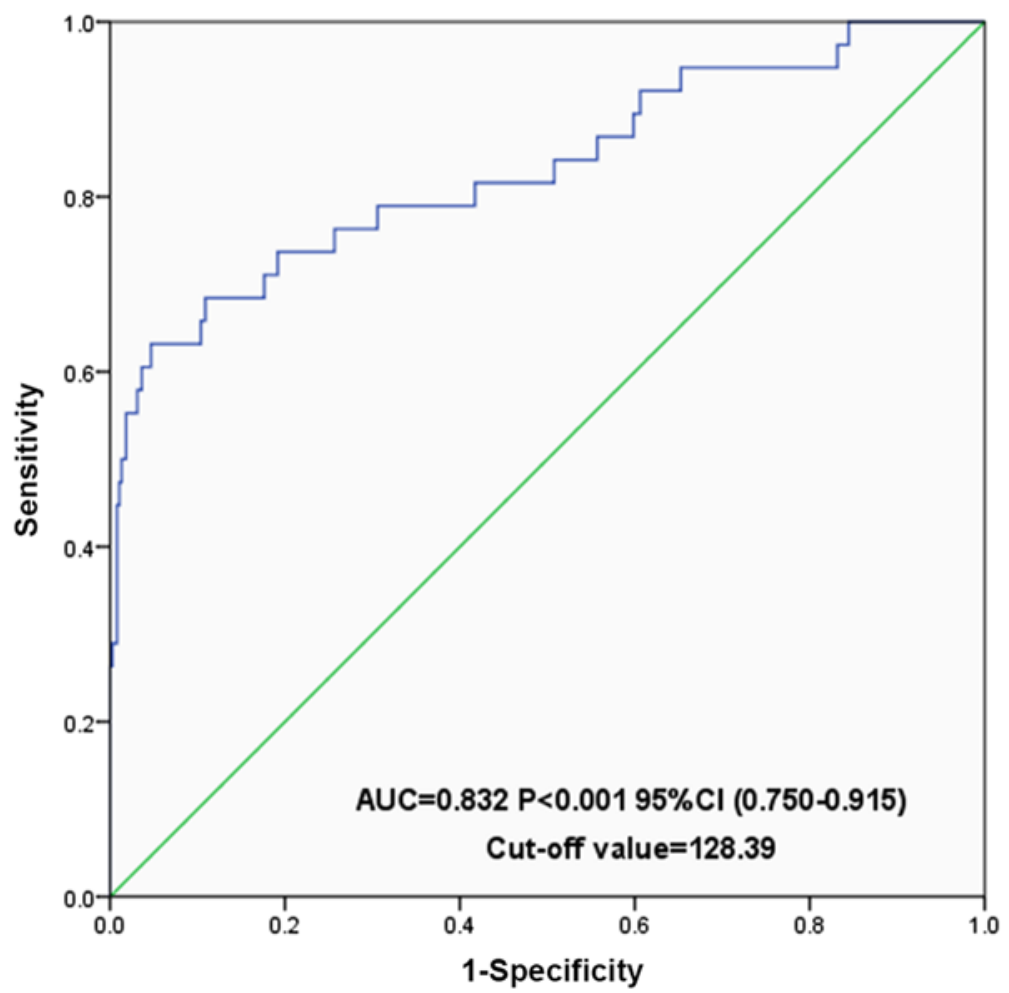

Figure 3. ROC curve for the fibrinogen-to-albumin ratio in predicting 5-year mortality. ROC, receiver operating characteristic; AUC, area under the ROC curve.

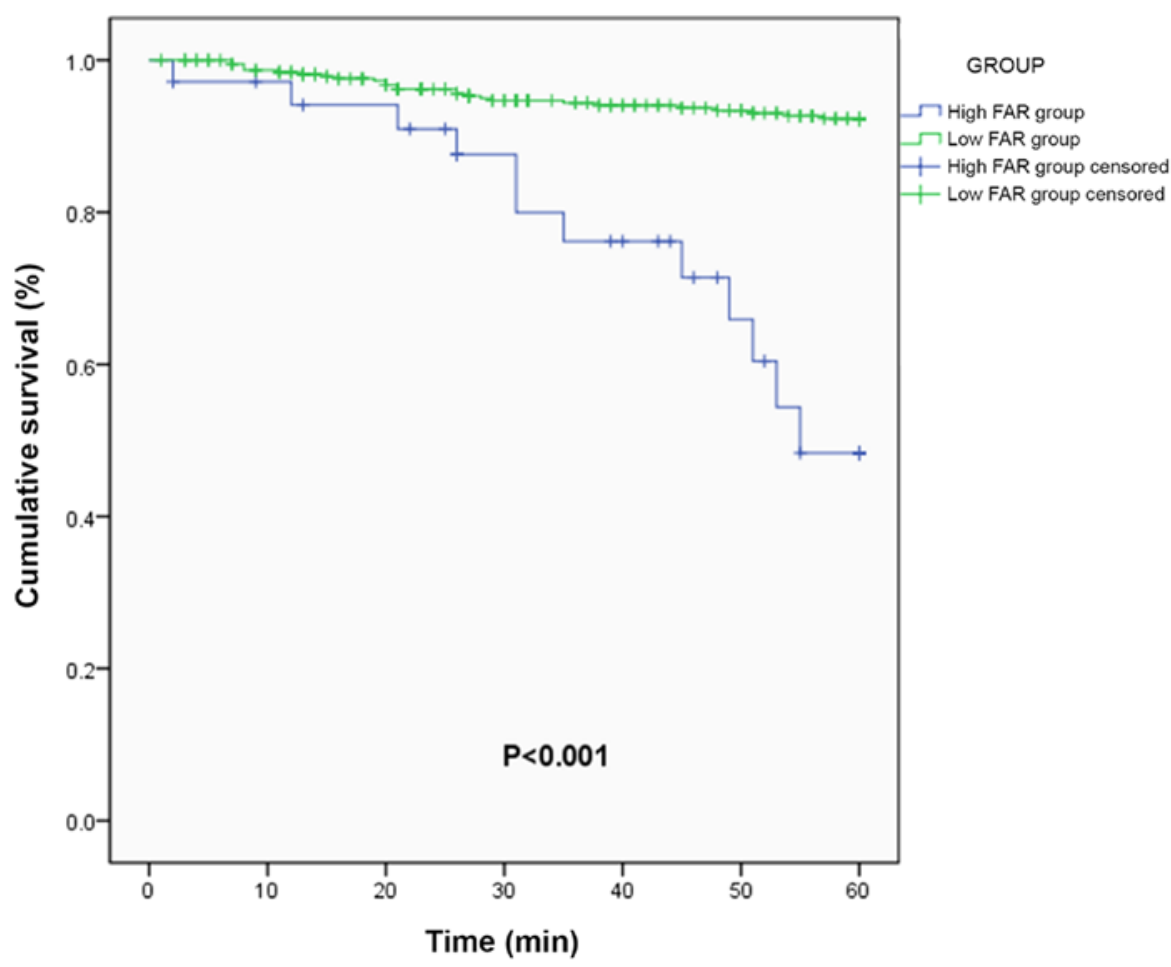

Figure 4. Kaplan-Meier survival curves for the primary endpoint for the high and the low FAR group (cut-off value, 128.4). FAR, fibrinogen-to-albumin ratio; m, months.

group had an unfavorable survival curve and a significantly lower survival rate $(\mathrm{P}<0.001$; Fig. 4). Univariate Cox regression found that FAR was associated with worse prognosis (HR, 1.031; 95\% CI, 1.023-1.040; $\mathrm{P}<0.001$ ). Multivariate
Cox regression analysis demonstrated that the FAR was an independent risk factor for the all-cause death (HR, 1.029; 95\% CI, 1.020-1.037; P<0.001; Table III), adjusted by age, heart failure, hs-CRP, and hypertension. 
Table III. Multivariate Cox regression analysis of factors influencing the primary endpoint.

\begin{tabular}{lcccrrr}
\hline Risk factor & B & SE & Wald & P-value & HR & 95\% CI \\
\hline Age $^{\mathrm{a}}$ & 0.043 & 0.013 & 10.669 & 0.001 & 1.044 & $1.017-1.071$ \\
Heart failure $^{\mathrm{b}}$ & 0.708 & 0.326 & 4.723 & 0.030 & 2.030 & $1.072-3.843$ \\
hs-CRP $^{\mathrm{a}}$ & 0.030 & 0.017 & 3.090 & 0.079 & 1.031 & $0.997-1.066$ \\
Hypertension $^{\mathrm{b}}$ & 0.907 & 0.339 & 7.146 & 0.008 & 2.477 & $1.274-4.816$ \\
FAR $^{\mathrm{a}}$ & 0.029 & 0.004 & 45.612 & $<0.001$ & 1.029 & $1.020-1.037$ \\
\hline
\end{tabular}

${ }^{\mathrm{a} C}$ Continuous variables. ${ }^{\mathrm{b}}$ Categorical variables. hs-CRP, high-sensitivity C-reactive protein; FAR, fibrinogen-to-albumin ratio; SE, standard error; HR, hazard ratio.

Table IV. Cox regression analysis of secondary endpoints for the high FAR group vs. low FAR group.

\begin{tabular}{|c|c|c|c|c|}
\hline \multirow[b]{2}{*}{ Factor } & \multicolumn{2}{|c|}{ Unadjusted } & \multicolumn{2}{|c|}{ Adjusted $^{\mathrm{a}}$} \\
\hline & $\mathrm{HR}(95 \% \mathrm{CI})$ & P-value & $\mathrm{HR}(95 \% \mathrm{CI})$ & P-value \\
\hline \multicolumn{5}{|l|}{ Secondary endpoint } \\
\hline Myocardial infarction & $1.79(3.75-7.87)$ & $<0.001$ & $1.62(3.49-7.52)$ & 0.001 \\
\hline Stroke & $0.21(1.67-13.18)$ & 0.628 & $0.23(1.95-16.34)$ & 0.537 \\
\hline Emergent revascularization & $0.71(2.03-5.82)$ & 0.186 & $0.81(2.4-7.15)$ & 0.115 \\
\hline Readmission due to heart failure & $0.15(1.14-8.77)$ & 0.901 & $0.13(1.05-8.41)$ & 0.961 \\
\hline Total secondary end-points & $1.48(2.56-4.45)$ & 0.001 & $1.48(2.62-4.63)$ & 0.001 \\
\hline
\end{tabular}

${ }^{a} \mathrm{HR}$ adjusted for covariates of age, heart failure, hypertension and hs-CRP. hs-CRP, high-sensitivity C-reactive protein; FAR, fibrinogen-to-albumin ratio; HR, hazard ratio.

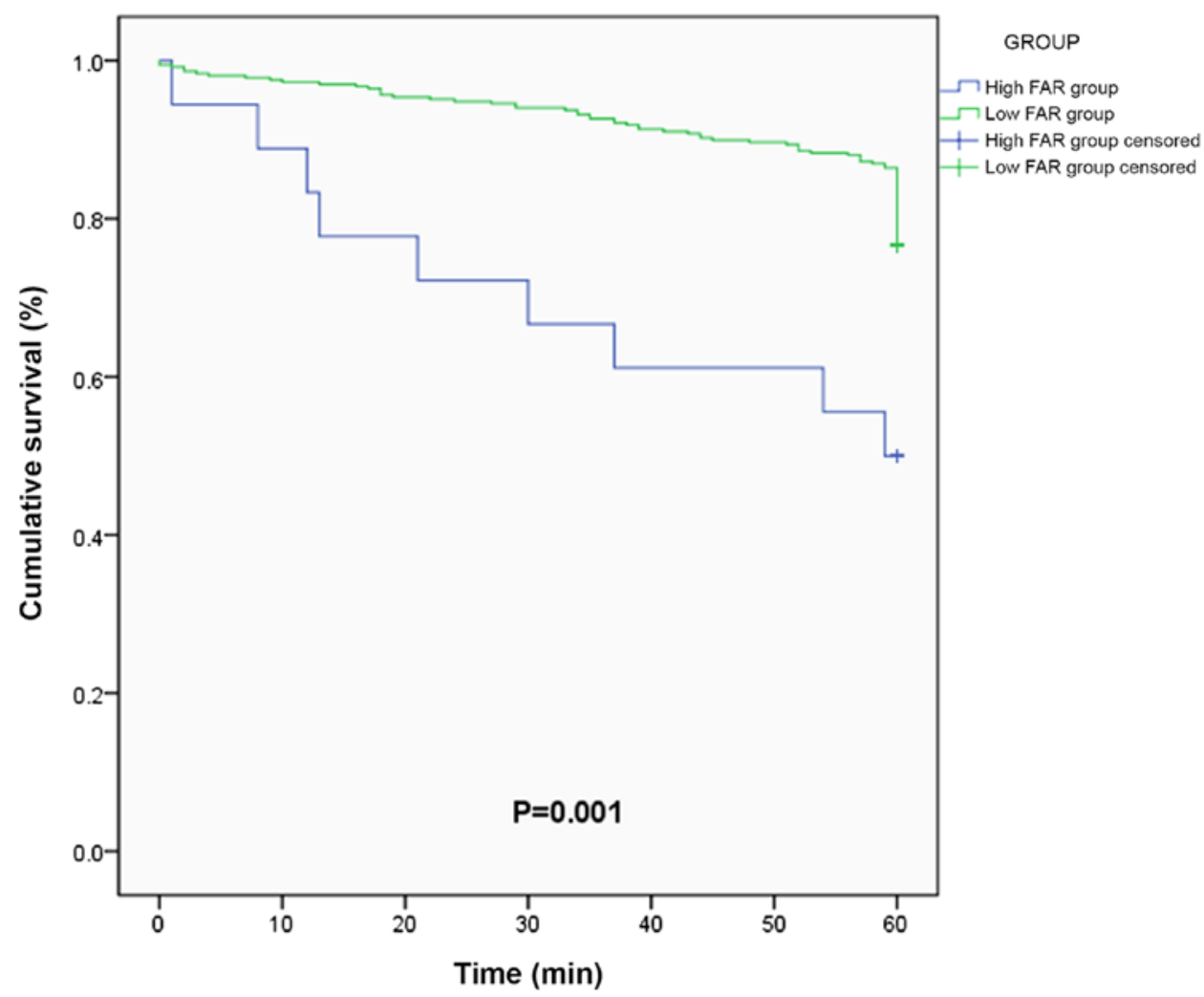

Figure 5. Kaplan-Meier curves for secondary endpoints for the high and the low FAR group (cut-off value, 128.4). FAR, fibrinogen-to-albumin ratio; m, months. 
Secondary endpoints. The secondary endpoints were also compared between the high FAR and the low FAR groups, which were MACE including the recurrent MI, stroke, unscheduled revascularization and rehospitalization due to heart failure (Fig. 5). The high FAR group had a significantly higher incidence of secondary endpoints $(\mathrm{P}=0.001)$. The multivariate Cox regression analysis demonstrated that the FAR was an independent risk factor for total MACE (HR, $1.48 ; 95 \%$ CI, 2.62-4.63; $\mathrm{P}=0.001$ ), even adjusted by age, heart failure, hypertension and hs-CRP in multivariate analysis (Table IV). Subgroup analysis for individual MACE indicated that only MI was significantly associated with the FAR (HR, 1.62; 95\% CI, 3.49-7.52; $\mathrm{P}<0.001$ ), while the other three endpoints were not (all $\mathrm{P}>0.05$ ).

\section{Discussion}

Abundant pieces of evidence have linked the FAR with the severity and prognosis of CAD and demonstrated its value in risk stratification and prediction of the prognosis of STEMI $(10,16-18)$. The present study not only investigated the association between the FAR and the SYNTAX score but explored the importance of the FAR in predicting the long-term outcomes for the patients with STEMI and multivessel disease. It was revealed that the FAR exhibited a linear correlation with the SYNTAX score, indicating its potential in assessing the severity. The observational cohort study proved that the FAR had potential value in predicting long-term outcomes. Of note, Cox regression analysis indicated no significant association between the FAR and stroke, unscheduled revascularization or rehospitalization for heart failure.

For patients with STEMI and multivessel disease, the prognosis remains unsatisfying. It was reported that patients with STEMI and multivessel CAD had poorer long-term mortality and a higher incidence of MACE (19). Different treatment strategies have been developed with controversies regarding their usefulness continuing. Previous observational and small randomized controlled trials have demonstrated conflicting results regarding the optimal treatment strategy (20). Specifically, complete vs. culprit-only revascularization has been compared. A review summarized current evidence on the different treatment options and concluded that the optimal revascularization strategy remains uncertain, while interventional cardiologists should determine the optimal reperfusion strategy based on concrete factors, including clinical characteristics, disease severity and complexity of lesions (1). A simple, cost-effective and sufficient risk assessment approach is required to predict the prognosis and direct the clinical strategy.

The SYNTAX scoring system is ubiquitously applied worldwide to assess the severity of CAD. Certain studies have also explored its value in prognosis prediction. Braga et al (4) reported on a retrospective cohort study on 535 patients with STEMI undergoing primary-PCI, who were divided into three groups according to their residual SYNTAX score (rSS): Complete revascularization $(\mathrm{rSS}=0 ; \mathrm{n}=198)$, reasonable incomplete revascularization $(0<\mathrm{rSS}<8 ; \mathrm{n}=238)$ and incomplete revascularization $(\mathrm{rSS} \geq 8 ; \mathrm{n}=99)$. The study suggested that rSS was positively correlated with in-hospital mortality and MACE (4). In the present study, the association between the SYNTAX score and FAR was analyzed, indicating a linear correlation between them. Compared with the SYNTAX score, the FAR has the advantages of simplicity, efficiency and sufficiency. A higher fibrinogen and lower albumin concentration are associated with worse outcomes for patients with CAD $(21,22)$. As the combination of fibrinogen and albumin as a ratio, the FAR is more sensitive and accurate as a stratification marker for CAD. Cetin et al (16) investigated the predictive value of the FAR in ACS, which demonstrated that FAR may be used for the prediction of MACE in patients with ACS who have undergone PCI. Another study indicated that the baseline FAR was independently and significantly associated with angiographic no-reflow and short-term mortality in patients with ACS (18). Different from previous studies, the present study focused on long-term outcomes of patients with STEMI and multivessel disease using a prospective cohort design. The present and previous studies have indicated that the FAR is a biomarker reflecting the systemic inflammation level and associated with the severity of CAD. However, it should be emphasized that the FAR is a valuable biomarker in the prognosis prediction of multiple tumors, including esophageal squamous cell carcinoma, non-small cell lung cancer, colorectal cancer and gallbladder cancer. As a result, there is a possibility that the association between the FAR and the prognosis of patients with STEMI may be falsely positive, which requires further validation.

Several limitations of the present study must be noted. First, only one PCI strategy was selected in the present study, which limited the sample size and brought about certain bias. Furthermore, the long-term prognosis was affected by the compliance of patients, which was not recorded or considered in the present study. Finally, a comparison between the FAR and other markers, including hs-CRP, albumin and fibrinogen alone, may be helpful.

In conclusion, the FAR was correlated with long-term mortality and the incidence of MACE in patients with STEMI and multivessel disease, which may indicate the potential of the FAR to serve as a biomarker.

\section{Acknowledgements}

Not applicable.

\section{Funding}

No funding was received.

\section{Availability of data and materials}

The datasets used and/or analyzed during the current study are available from the corresponding author on reasonable request.

\section{Authors' contributions}

GL and GXC designed and led the current study. MLL, HG, WNF and CMF collected and analyzed the patient data. GL and HG wrote the manuscript and WNF revised it. All authors read and approved the final manuscript. 


\section{Ethics approval and consent to participate}

The present study was approved by the Medical Ethics Committee of Xianyang Central Hospital (Xianyang, China). The patients provided written informed consent regarding the use of their data.

\section{Patient consent for publication}

Not applicable.

\section{Competing interests}

The authors declare that they have no competing interests.

\section{References}

1. Vogel B, Mehta SR and Mehran R: Reperfusion strategies in acute myocardial infarction and multivessel disease. Nat Rev Cardiol 14: 665-678, 2017.

2. de Waha S, Eitel I, Desch S, Fuernau G, Pöss J, Schuler G and Thiele H: Impact of multivessel coronary artery disease on reperfusion success in patients with ST-elevation myocardial infarction: A substudy of the AIDA STEMI trial. Eur Heart J Acute Cardiovas Care 6: 592-600, 2017.

3. Bates ER, Tamis-Holland JE, Bittl JA, O'Gara PT and Levine GN PCI strategies in patients with ST-segment elevation myocardial infarction and multivessel coronary artery disease. J Am Coll Cardiol 68: 1066-1081, 2016.

4. Braga CG, Cid-Alvarez AB, Dieguez AR, Alarez BA, Otero DL, Sánchez RO, Pena XS, Salvado VG, Trillo-Nouche R and González-Juanatey JR: Prognostic impact of residual SYNTAX score in patients with ST-elevation myocardial infarction and multivessel disease: Analysis of an 8-year all-comers registry. Int J Cardiol 243: 21-26, 2017.

5. Wilhelmsen L, Svardsudd K, Korsan-Bengtsen K, Larsson B, Welin L and Tibblin G: Fibrinogen as a risk factor for stroke and myocardial infarction. N Engl J Med 311: 501-505, 1984.

6. Nelson JJ, Liao D, Sharrett AR, Folsom AR, Chambless LE, Shahar E, Szklo M, Eckfeldt J and Heiss G: Serum albumin level as a predictor of incident coronary heart disease: The atherosclerosis risk in communities (ARIC) study. Am J Epidemiol 151: 468-477, 2000.

7. Kijima T, Arigami T, Uchikado Y, Uenosono Y, Kita Y, Owaki T, Mori S, Kurahara H, Kijima Y and Okumura H: Combined fibrinogen and neutrophil-lymphocyte ratio as a prognostic marker of advanced esophageal squamous cell carcinoma. Cancer Med 108: 193-199, 2017.

8. Li SQ, Jiang YH, Lin J, Zhang J, Sun F, Gao QF, Zhang L, Chen QG, Wang XZ and Ying HQ: Albumin-to-fibrinogen ratio as a promising biomarker to predict clinical outcome of non-smal cell lung cancer individuals. Cancer Med 7: 1221-1231, 2018.

9. Sun F, Tan YA, Gao QF, Li SQ, Zhang J, Chen QG, Jiang YH, Zhang L, Ying HQ and Wang XZ: Circulating fibrinogen to pre-albumin ratio is a promising biomarker for diagnosis of colorectal cancer. J Clin Lab Anal 33: e22635, 2019.

10. Xu WY, Zhang HH, Xiong JP, Yang XB, Bai Y, Lin JZ, Long JY, Zheng YC, Zhao HT and Sang XT: Prognostic significance of the fibrinogen-to-albumin ratio in gallbladder cancer patients. World J Gastroenterol 24: 3281-3292, 2018.
11. Karahan O, Acet H, Ertas F, Tezcan O, Caliskan A, Demir M, Kaya AF, Demirtaş S, Çevik MU and Yavuz C: The relationship between fibrinogen to albumin ratio and severity of coronary artery disease in patients with STEMI. Am J Emerg Med 34: 1037-1042, 2016.

12. Task Force on the management of ST-segment elevation acute myocardial infarction of the European Society of Cardiology (ESC); Steg PG, James SK, Atar D, Badano LP, Blömstrom-Lundqvist C, Borger MA, Di Mario C, Dickstein K, Ducrocq G, et al: ESC Guidelines for the management of acute myocardial infarction in patients presenting with ST-segment elevation. Eur Heart J 33: 2569-2619, 2012.

13. Ibanez B, James S, Agewall S, Antunes MJ, Bucciarelli-Ducci C, Bueno H, Caforio ALP, Crea F, Goudevenos JA, Halvorsen S, et al: 2017 ESC Guidelines for the management of acute myocardial infarction in patients presenting with ST-segment elevation: The task force for the management of acute myocardial infarction in patients presenting with ST-segment elevation of the European Society of Cardiology (ESC). Eur Heart J 39: 119-177, 2018.

14. Lee JM, Rhee TM, Kim HK, Hwang D, Lee SH, Choi KH, Kim J, Park TK, Yang JH, Song YB, et al: Comparison of long-term clinical outcome between multivessel percutaneous coronary intervention versus infarct-related artery-only revascularization for patients with St-segment-elevation myocardial infarction with cardiogenic shock. J Am Heart Assoc 8: e013870, 2019.

15. Bohula EA, Morrow DA, Giugliano RP, Blazing MA, He P, Park JG, Murphy SA, White JA, Kesaniemi YA, Pedersen TR, et al: Atherothrombotic risk stratification and ezetimibe for secondary prevention. J Am Coll Cardiol 69: 911-921, 2017.

16. Cetin M, Erdoğan T, Kırıs T, Özer S, Yılmaz AS, Durak H, Aykan AÇ and Şatıroğlu Ö: Predictive value of fibrinogen-to-albumin ratio in acute coronary syndrome. Herz: Aug 6, 2019 (Epub ahead of print).

17. Kayapinar O, Ozde C and Kaya A: Relationship between the reciprocal change in inflammation-related biomarkers (fibrinogen-to-albumin and hsCRP-to-albumin ratios) and the presence and severity of coronary slow flow. Clin Appl Thromb Hemost 25: 1076029619835383, 2019.

18. Zhao Y, Yang J, Ji Y, Wang S, Wang T, Wang F and Tang J: Usefulness of fibrinogen-to-albumin ratio to predict no-reflow and short-term prognosis in patients with ST-segment elevation myocardial infarction undergoing primary percutaneous coronary intervention. Heart Vessels 34: 1600-1607, 2019.

19. Tarantini G, D'Amico G, Brener SJ, Tellaroli P, Basile M, Schiavo A, Mojoli M, Fraccaro C, Marchese A, Musumeci G and Stone GW: Survival after varying revascularization strategies in patients with ST-segment elevation myocardial infarction and multivessel coronary artery disease: A pairwise and network meta-analysis. JACC Cardiovas Interv 9: 1765-1776, 2016.

20. Di Pasquale G, Filippini E, Pavesi PC, Tortorici G, Casella G and Sangiorgio P: Complete versus culprit-only revascularization in ST-elevation myocardial infarction and multivessel disease. Int Emerg Med 11: 499-506, 2016.

21. O'Donoghue ML, Morrow DA, Cannon CP, Jarolim P, Desai NR, Sherwood MW, Murphy SA, Gerszten RE and Sabatine MS: Multimarker risk stratification in patients with acute myocardial infarction. J Am Heart Assoc 5: pii: e002586, 2016.

22. Sanchez PL, Morinigo JL, Pabon P, Martin F, Piedra I, Palacios IF and Martin-Luengo C: Prognostic relations between inflammatory markers and mortality in diabetic patients with non-ST elevation acute coronary syndrome. Heart 90: 264-269, 2004.

This work is licensed under a Creative Commons Attribution-NonCommercial-NoDerivatives 4.0 International (CC BY-NC-ND 4.0) License. 